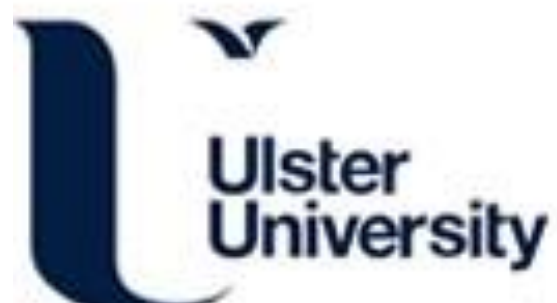

\section{Accurate and Robust Object SLAM with 3D Quadric Landmark Reconstruction in Outdoors}

Tian, R., Zhang, Y., Feng, Y., Yang, L., Cao, Z., Coleman, S., \& Kerr, D. (2022). Accurate and Robust Object SLAM with 3D Quadric Landmark Reconstruction in Outdoors. IEEE Robotics and Automation Letters, 7(2), 1534-1541. https://doi.org/10.1109/LRA.2021.3137896

Link to publication record in Ulster University Research Portal

Published in:

IEEE Robotics and Automation Letters

Publication Status:

Published (in print/issue): 30/04/2022

DOI:

10.1109/LRA.2021.3137896

\section{Document Version}

Author Accepted version

\section{General rights}

Copyright for the publications made accessible via Ulster University's Research Portal is retained by the author(s) and / or other copyright owners and it is a condition of accessing these publications that users recognise and abide by the legal requirements associated with these rights.

\section{Take down policy}

The Research Portal is Ulster University's institutional repository that provides access to Ulster's research outputs. Every effort has been made to ensure that content in the Research Portal does not infringe any person's rights, or applicable UK laws. If you discover content in the Research Portal that you believe breaches copyright or violates any law, please contact pure-support@ulster.ac.uk. 


\title{
Accurate and Robust Object SLAM with 3D Quadric Landmark Reconstruction in Outdoor Environment
}

\author{
Rui Tian ${ }^{1}$, Yunzhou Zhang ${ }^{1 *}$, Yonghui Feng ${ }^{1}$, Linghao Yang ${ }^{1}$, \\ Zhenzhong $\mathrm{Cao}^{1}$, Sonya Coleman ${ }^{2}$, Dermot Kerr $^{2}$
}

\begin{abstract}
Object-oriented SLAM is a popular technology in autonomous driving and robotics. In this paper, we propose a stereo visual SLAM with a robust quadric landmark representation method. The system consists of four components, including deep learning detection, quadric landmark initialization, object data association and object pose optimization. State-of-the-art quadric-based SLAM algorithms always face observation related problems and are sensitive to observation noise, which limits their application in outdoor scenes. To solve this problem, we propose a quadric initialization method based on the separation of the quadric parameters method, which improves the robustness to observation noise. The sufficient object data association algorithm and object-oriented optimization with multiple cues enables a highly accurate object pose estimation that is robust to local observations. Experimental results show that the proposed system is more robust to observation noise and significantly outperforms current stateof-the-art methods in outdoor environments. In addition, the proposed system demonstrates real-time performance.
\end{abstract}

\section{INTRODUCTION}

Simultaneous Localization and Mapping (SLAM) is a fundamental technique in order for robots to perceive the environment. When compared with classic SLAM methods that use only the geometry of the scene, object-based SLAM has recently focused on creating maps with both geometry and high-level semantic objects within the environment [1]-[10]. This semantically-enriched information can help robots with target-oriented tasks like obstacle avoidance, robust relocalization and human-robot interaction. The improvement in the accuracy of semantic information acquisition, driven by deep learning networks [11]-[13], has led to the increasing introduction of object detection and semantic segmentation into visual SLAM systems to build semantically enriched maps and enhance the perception ability of robots.

Accurate object representation is a key issue in objectoriented SLAM research and 3D object models [14], cubic boxes [3]-[5] and ellipsoids [6]-[8] are among common methods utilized for object representation. Prior work like [4] and [5] use the cubic box to represent the object, where the pose of the cubic box can be estimated by vanishing

\footnotetext{
* The corresponding author of this paper.

${ }^{1}$ Rui Tian, Yunzhou Zhang, Yonghui Feng, Linghao Yang and Zhenzhong Cao are with College of Information Science and Engineering, Northeastern University, Shenyang 110819, China (Email: zhangyunzhou@mail. neu.edu.cn).

${ }^{2}$ Sonya Coleman and Dermot Kerr are with School of Computing, Engineering and Intelligent Systems, Ulster University, N. Ireland, UK.

This work was supported by National Natural Science Foundation of China (No. 61973066, 61471110) and the Distinguished Creative Talent Program of Liaoning Colleges and Universities (LR2019027).
}
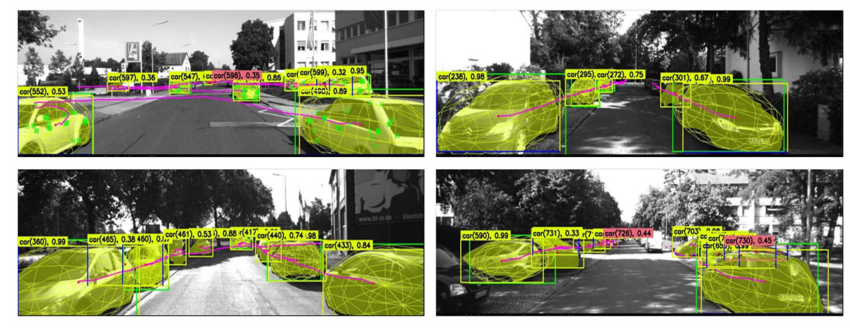

Fig. 1. The proposed method uses 3D quadric landmarks to build the object map in outdoor environments. Yellow quadrics illustrate the accuracy of orientation and shape of the estimated ellipsoids when projected onto the image frame. Object ID is marked, and the magenta lines show the center of the ellipsoids in previous frames when projected onto the image frame, indicating the accuracy of the object data association.

points and rotation sampling. Compared with the cubic box, the ellipsoid can also accurately represent the position, orientation and size of the object and has a more concise mathematical representation [6]. In projective geometry the quadric can be represented by a symmetric matrix [15] where the compact perspective projection model and the closed surfaces of ellipsoids are meaningful for object landmarks.

The accuracy and robustness of current quadric-based SLAM are not ideal, especially the quadric initialization process, which is limited by the parameter coupling of the direct linear solution method [6] or the necessity for point cloud fitting [7], [9]. QuadricSLAM [6] is a recently proposed object-oriented SLAM system that represents objects as quadrics; a dual quadric observation model based on the object detection is proposed. However, the closed-form constrained dual quadric parameterization and the lack of observation angles under the planar trajectory of the mobile vehicle make the initialization of the quadric difficult and sensitive to observation noise. In [9], multiple constraints combined with points, surface and quadrics are used in the optimization framework, but the prior shape of the object is estimated based on deep learning which incurs a high computational complexity and is not robust. In [2], the texture plane and shape prior constraints are added to the quadric estimation which solves the problem of poor estimation performance when the observation angles change in road driving scenes. However, the assumption that the texture plane is parallel to the image plane during quadric initialization causes the estimation to be sensitive to noise.

In addition, in prior work such as [6], [16], data association methods have been proposed although they are typically not robust to outdoor scenes. Dynamic objects in outdoor scenes like moving cars and persons are a challenge for quadric estimation since false object associations will lead to false 
quadric initialization results.

To solve the aforementioned problems, we propose a robust and accurate quadric landmark initialization based on a method for separating of quadric parameters (SQP) and an object data association (ODA) algorithm in outdoor scenes. The robustness of SQP to observation noise is improved by independently estimating the quadric centroid translation and the yaw rotation constraint which is satisfied for autonomous vehicles in road planes in most cases. Then, an ellipsoid with improved accuracy can be obtained by a nonlinear optimizer combining the detection observation error, the texture plane error and the prior size constraint. In terms of data association, we propose a multiple-cues algorithm combined with the Hungarian assignment algorithm [17] which improves the robustness of object pose estimation.

We demonstrate the performance of the proposed system in both a simulation environment and using the KITTI Raw Data [18] datasets. The experimental results show that the proposed system is more robust to observation noise than other existing methods and improves the accuracy of the position, orientation and size of the object estimation in the outdoor environment.

\section{The main contributions of this work are:}

- To effectively overcome the observation noise, we propose an accurate and robust quadric landmark initialization method based on the SQP algorithm by separating of translation and rotation of quadric centroids.

- We proposed an ODA algorithm that combines the semantic inliers distribution, Kalman-based motion prediction, and ellipsoidal projection to achieve accurate data association and jointly estimates the camera pose and the quadric landmarks of objects.

- Based on the proposed algorithms, we implement realtime stereo visual SLAM with accurate and robust ellipsoids representing objects, aiming to build an objectoriented and semantically-enhanced map for outdoor navigation.

\section{SySTEM OVERVIEW}

\section{A. Mathematical Representation of a Quadric Model}

For convenience of description, the notations used in this paper are as follows:

- $(\cdot)_{w}$ is the world coordinate, $(\cdot)_{c}$ is the camera coordinate, $(\cdot)_{r}$ is the reference camera coordinate of the object, and $(\cdot)_{q}$ is the quadric center frame.

- $K$ - The intrinsic matrix of a pinhole camera model.

- $T_{c w} \in R^{4 \times 4}$ - The transformation from world frame to camera frame, which is composed of a rotation $R_{c w} \in$ $R^{3 \times 3}$ and a translation $t_{c w} \in R^{3 \times 1}$.

- $P=K T$ - The camera projection matrix that contains intrinsic and extrinsic camera parameters.

- $B=\left[x_{\min }, y_{\min }, x_{\max }, y_{\max }\right]^{T}$ - The 2D object detection bounding box (BBox).

- $L=\left\{l_{k}\right\}$ - The set of four lines of 2D object detection BBox.

- $M$ is the segmentation instance mask, $D$ is the detection instance, $O$ is the object instance.

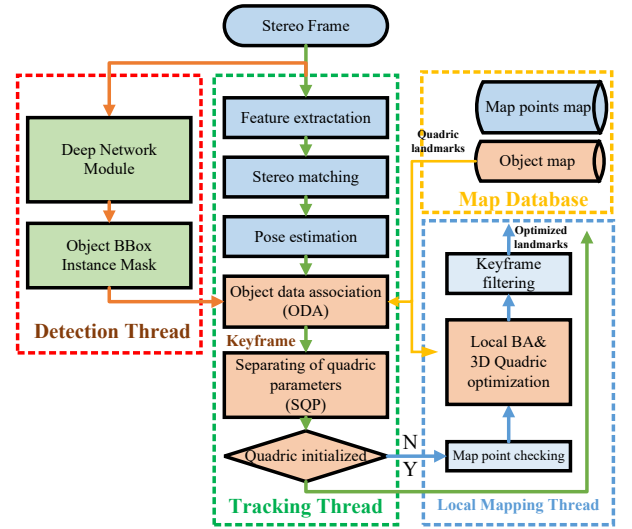

Fig. 2. Overview of our proposed system, there are two key modules: 1) The detection thread takes images and acquires semantic and detection results. 2) The tracking thread initializes quadrics with the SQP method and the ODA associates detected objects with the mapped objects for further optimization. The Local mapping thread jointly optimizes quadrics and pose. Finally, the object map is stored with ellipsoid landmarks representations.

- $D_{j}^{i}$ represent the detected object instance $D_{j}$ that is assigned to the object $O_{i}, \operatorname{cls}(D)$.

- $\operatorname{In}(B, x)$ - The checking of image points $x$ that are located in the $B$ detection box.

- $Q \in R_{4 \times 4}$ - The quadric matrix in 3D space and $Q^{*} \in$ $R_{4 \times 4}$ is denoted as the dual quadric matrix.

- $\Pi_{k}=\left[\pi_{1}, \pi_{2}, \pi_{3}, \pi_{4}\right]^{T}$ - The 3-D plane surface in homogeneous coordinate and all quadric plane fulfil $\Pi_{k}^{T} Q^{*} \Pi_{k}=0$ and $\Pi_{k}=P^{T} l_{k}$.

- $q=\left[a_{x}, a_{y}, a_{z}, t_{x}, t_{y}, t_{z}, \theta_{x}, \theta_{y}, \theta_{z}\right]^{T}$ - The 9-D vector representing the attributes of the quadric, including axial length, translation and rotation.

When a dual quadric is projected onto an image plane, it creates a dual conic, following the rule $C^{*}=P Q^{*} P^{T}$. For more specific properties of the quadric, please refer to [6].

\section{B. System Architecture}

The proposed system is shown in Fig.2. We implement our algorithms on the basis of ORB-SLAM3 [19], and a stereo camera is used to obtain a metric scale of the estimated trajectory for the autonomous driving scene to avoid scale ambiguity caused by monocular SLAM [20]. However, we also highlight that our method can be used for monocular SLAM. There are two key modules, the visual SLAM module and the detection module. The visual SLAM module consists of parallel threads, including the tracking thread and the local mapping thread. Finally, the camera pose is estimated and a semantically-enhanced object map is also stored in the map database.

(1) The detection thread uses YOLOACT [11] to acquire semantic information from the left images of the stereo pair. The output results are object detection BBoxes and the instance segmentation masks.

(2) The tracking thread takes images and estimates the camera pose from consecutive frames. Meanwhile, the thread waits for the detection instances and associates them with the existing objects in the object map database or decides whether to insert a keyframe and initialize a new object. In addition, if the current frame is a keyframe and 
the observations satisfy the quadric initialization condition, the SQP algorithm is used for robust and accurate quadric initialization.

(3) The local mapping thread optimizes the map points, ellipsoids and poses of keyframes with the local bundle adjustment (BA). In addition, when the objects are observed by newly inserted keyframes, the new observation can be added to the object optimizer for nonlinear optimization of the ellipsoidal representation of objects.

(4) The map Database stores the final maps, including the geometry information of map points and the object map with ellipsoids.

\section{SEPARATING OF QUADRIC PARAMETERS INITIALIZATION ALGORITHM}

\section{A. Independent estimation of Quadric Central Translation}

We present the mathematical analysis of the dual quadric parameters to illustrate the effect of the translation component on the estimation of rotation and shape. The dual form parameters of the ellipsoid can be decomposed by eigendecomposition in the reference camera coordinates of the object:

$$
\begin{aligned}
& Q_{r}^{*}=T_{r q} Q_{q}^{*} T_{r q}^{T} \\
& =\left[\begin{array}{cc}
R_{r q} & t_{r q} \\
0^{T} & 1
\end{array}\right]\left[\begin{array}{cc}
D & 0 \\
0^{T} & -1
\end{array}\right]\left[\begin{array}{cc}
R_{r q}^{T} & 0 \\
t_{r q}^{T} & 1
\end{array}\right] \\
& =\left[\begin{array}{cc}
R_{r q} D R_{r q}^{T}-t_{r q} t_{r q}^{T} & -t_{r q} \\
-t_{r q}^{T} & -1
\end{array}\right] \\
& =\left[\begin{array}{cc}
Q_{r 33}^{*} & -t_{r q} \\
-t_{r q}^{T} & -1
\end{array}\right]
\end{aligned}
$$

where $D \in R^{3 \times 3}$ is the diagonal matrix composed of the squares of the quadric axial lengths, and $t_{r q} \in R^{3 \times 1}$ is the quadric centroid translation in the reference camera coordinates. The parameters of the block matrix $Q_{r}^{*} \in R^{3 \times 3}$ couple the rotation and translation of the quadric. Since the length of the quadric centroid translation is much larger than that of the rotation and axes, small errors in the estimation of the quadric centroid translation have a significant impact on the accurate estimation of the dual quadric matrix, which is why QuadricSLAM [6] is sensitive to observation noise, which often occurs when the viewing angle is limited and the detection observation occluded.

We can also see from Eq.(1) that the translation parameters are independent in dual form elements of $q_{14}^{*}, q_{24}^{*}, q_{34}^{*}$. Therefore we estimate the translation component parameters $t_{r q} \in R^{3 \times 1}$ independently to eliminate the effect of coupling parameters, a key aspect of our approach. We triangulate the center of the 2D detection box $x_{b c}^{i}$ and obtain the triangulation map point $\hat{t}_{r q}$, which is almost close with the quadric center $t_{r q}$ in outdoor scenes. This assumption is proved by experiments in VI-A. Observations of two or more frames of detection centers form the overdetermined equation to solve $t_{r q}$,

$$
\left[\begin{array}{c}
P_{c}\left[1,{ }^{*}\right] T_{w r}-x_{b c}^{i}[1] \cdot P_{c}\left[3,{ }^{*}\right] T_{w r} \\
P_{c}\left[2,{ }^{*}\right] T_{w r}-x_{b c}^{i}[2] \cdot P_{c}\left[3,{ }^{*}\right] T_{w r}
\end{array}\right] t_{r q}=0
$$

where, $x_{b c}^{i}[i]$ is the $i$-th element of 2D BBox center, $P_{c}[i, *]$ is the $i$-th row of the projection matrix $P_{c}$.

We mentioned that the translation component of the quadric can be estimated independently with fewer constraints by using Eq.(2) when the observations occluded or limited viewing angles.

\section{B. Estimation of Quadric Rotation and Axial Length}

The rotation and quadric axial lengths are considered after the quadric centroid translation has been estimated independently. We assume that the ellipsoid of the object, such as an autonomous vehicle or robot, is under the constraint of yaw rotation, while the pitch and roll are constant at zero. This is satisfied for autonomous vehicles on the road in outdoor scenes. Therefore, we can replace the rotation matrix in Eq.(1) by:

$$
\begin{aligned}
& R_{r q}=\left[\begin{array}{ccc}
\cos \theta_{y} & 0 & \sin \theta_{y} \\
0 & 1 & 0 \\
-\sin \theta_{y} & 0 & \cos \theta_{y}
\end{array}\right]
\end{aligned}
$$

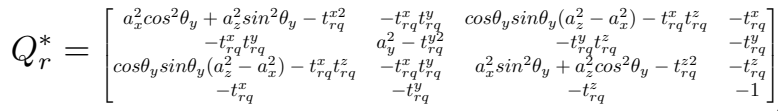

where, $t_{r q}^{x}, t_{r q}^{y}, t_{r q}^{z}$ are elements of the quadric centroid translation vector.

We can simplify the linear form in [6] by using the landmark BBox observations $B$ and the corresponding dual quadric planes $\Pi_{k}$ by substituting the $t_{r q}$.

$$
m_{k}=\left[\begin{array}{c}
\pi_{1}^{2} \\
2 \pi_{1} \pi_{3} \\
\pi_{2}^{2} \\
\pi_{3}^{2} \\
\pi_{4}^{2}+2 \pi_{1} \pi_{2} t_{r q}^{x} t_{r q}^{y}+2 \pi_{1} \pi_{4} t_{r q}^{x}+2 \pi_{2} \pi_{3} t_{r q}^{y} t_{r q}^{z} \\
+2 \pi_{2} \pi_{4} t_{r q}^{y}+2 \pi_{3} \pi_{4} t_{r q}^{z}
\end{array}\right]
$$

Where $N$ is the observation frames of the same associated detected object, the decoupled linear form of Eq.(5) can be solved by singular value decomposition (SVD) [6], where $q_{i j}$ is the remaining elements of the dual quadric $Q_{r}^{*}$ to be estimated.

Finally, the 9-D vector $q$ of the quadric with orientation, translation and axial lengths of the ellipsoid can be obtained by the estimated dual quadric matrix $Q_{r}^{*}$ :

$$
\begin{aligned}
\theta_{y} & =\arctan \left(2 Q_{3} /\left(Q_{8}-Q_{1}\right)\right) / 2 \\
a_{x} & =\sqrt{\left|Q_{1}-2 Q_{3}+Q_{8}\right| / 2} \\
a_{y} & =\sqrt{Q_{2}} \\
a_{z} & =\sqrt{\left|Q_{1}+2 Q_{3}+Q_{8}\right| / 2}
\end{aligned}
$$

where,

$$
\begin{aligned}
& Q_{1}=-\frac{q_{11}^{*}}{q_{44}^{*}}+t_{r q}^{x 2} \quad Q_{2}=-\frac{q_{22}^{*}}{q_{44}^{*}}+t_{r q}^{y 2} \\
& Q_{3}=-\frac{q_{13}^{*}}{q_{44}^{*}}+t_{r q}^{x} t_{r q}^{z} \quad Q_{8}=-\frac{q_{23}^{*}}{q_{44}^{*}}+t_{r q}^{z 2}
\end{aligned}
$$




\section{3D OBJECT OBSERVATION CONSTRAINTS OPTIMIZATION}

In the local mapping thread, we jointly optimize the quadrics and camera pose by using odometry factors and landmark factors combined with the observation of local keyframes. We define the set of detected objects as $\mathbb{D}$, and the set of mapped objects as $\mathbb{O}$. With the 3D map point landmarks $X$, the 2D feature observations $U=\left\{u_{i j}\right\}$, the observed instances $D_{j}$ and the associated mapped instance $O_{i}$, the joint observation error can be optimized with the following constraint:

$$
\begin{aligned}
T, X, q= & \underset{T, X}{\arg \min } \sum_{i} \sum_{j}\left\|f\left(T_{i}, X_{j}\right)-u_{i j}\right\|_{\Sigma_{i}}^{2}+ \\
& \underset{T, q}{\arg \min } \sum_{i} \sum_{j}\left\{\left\|e_{b}\left(B_{O_{i}}, B_{D_{j}}\right)\right\|_{\Sigma_{b}}^{2}+\right. \\
& \left.\left\|e_{a}\left(O_{i}\right)\right\|_{\Sigma_{a}}^{2}+\left\|e_{p}\left(\Pi_{D_{j}}, \Pi_{O_{i}}\right)\right\|_{\Sigma_{p}}^{2}\right\}
\end{aligned}
$$

Where $f($.$) is the camera projection model of 3D landmark$ $X_{j}$ observed in the reference frame with pose $T_{i}$ [19], and the Huber kernel is used to enhance the robustness of outliers, and the $L M$ algorithm is used to optimize the target cost function.

\section{A. The $2 D$ detection error}

The 2D detection error is used to calculate the distance error between the 2D object BBox $B_{O_{i}}^{k}$ transformed to the current frame by the camera projection matrix $P_{k}$ and the detected BBox $B_{D_{j}}$ in the $k^{t h}$ keyframe. Detection results near the edge of the image are ignored in order to eliminate the effect of occlusion, the variance is set experimentally by 2 pixels.

$$
e_{b}\left(B_{O_{i}}, B_{D_{j}}\right)=B_{O_{i}}^{k}-B_{D_{j}}
$$

\section{B. Prior axial length error}

The prior axial length constraint is used to mitigate the degenerate solution of an ambiguous scale of ellipsoid [2], which is calculated by the distance of the prior axial length $a_{O_{i}}^{\text {prior }}$ to the estimated quadric axial length $a_{O_{i}}$ with the certain class of object, e.g., the car class in our experiments, the prior car length is given experimentally by $[2,2,4]$ with the diagonal covariance matrix of 0.2 :

$$
e_{a}\left(O_{i}\right)=a_{O_{i}}^{\text {prior }}-a_{O_{i}}
$$

\section{Texture plane error}

Similar to the method proposed by [2], the texture plane error is obtained by the minimum distance between the fitted texture plane and the quadric landmark. The plane parameters of the texture plane is obtained by Delauney Triangulation of the object's map points with the normal vector $n_{D_{j}}$ and plane distance $Z_{D_{j}}$ of a texture plane $\Pi_{D_{j}}$. The variance on the texture plane $\Sigma_{p}$ is the empirical variance in the depth of the triangulated points, the texture plane distance error can be calculated as:

$$
e_{p}\left(\Pi_{D j}, \Pi_{O_{i}}\right)=Z_{D_{j}}-Z_{O_{i}}
$$

\section{The OBJect Data Association ALgorithm}

Multi-view geometry information is used for both object landmark initialization and optimization, while the object detection results are obtained by the single-frame image of current frame. Therefore, it is necessary to correctly associate the detected instance of the same object within the map. We propose the ODA algorithm to integrate information for data association. The Hungarian algorithm [17] is used to complete the assignment with the minimum distance error. Three different distance metrics are used for affinity functions to obtain $a_{i j}$, which is the element of the cost matrix $\mathbb{A}$. The $\alpha, \beta$ and $\gamma$ parameters are experimentally set to $0.8,1$, and 0.8 respectively.

$$
a_{i j}=\alpha a_{i j}^{p}+\beta a_{i j}^{g}+\gamma a_{i j}^{k}
$$

\section{A. Semantic Inliers Points Distance}

To overcome the overlap of the detection masks, we use Bi-directional Optical Flow (BODF) to track the keypoints within the detection mask $M_{j}$ from the last keyframe and obtain the keypoints set $\left\{x_{j}^{k}\right\}$. We calculate the ratio of inliers corresponding to the same object class, where size $\{$. calculates the element numbers of the set:

$$
a_{i j}^{p}=1-\frac{\operatorname{size}\left\{\operatorname{In}\left(M_{O_{i}}^{k}, X_{j}^{k}\right)\right\}}{\operatorname{size}\left\{X_{j}^{k}\right\}}
$$

\section{B. Intersection of Union Distance}

To calculate the intersection of union distance, we use the intersection ratio between the 2D quadric landmark projection BBox $B_{O i}$ of $O_{i}$ and the $2 \mathrm{D}$ detection result $B_{D_{j}}$ of the object instance $D_{j}$.

$$
a_{i j}^{g}=1-\frac{B_{O_{i}} \cap B_{D_{j}}}{B_{O_{i}} \cup B_{D_{j}}}
$$

\section{Prior Object Size Distance}

For each object instance $O_{i}$, the motion prediction method based on the Kalman filter [21] is adopted to predict the state of the detection in the image frame. The predicted 2D BBox of $D_{j}$ is denoted as $B_{D_{j}}^{a}$, the prior object size distance $a_{i j}^{k}$ is defined by:

$$
a_{i j}^{k}=1-\frac{B_{O_{i}}^{a} \cap B_{D_{j}}^{a}}{B_{O_{i}}^{a} \cup B_{D_{j}}^{a}}
$$

\section{EXPERIMENTS AND EVALUATION}

To evaluate the performance of our proposed method, we build an experimental simulation environment based on OpenGL ${ }^{1}$ to compare the quality of the constructed landmarks against other state-of-the-art techniques. We further use the KITTI Raw Data dataset [18] to evaluate the quality of the constructed quadric landmarks in a benchmark realworld dataset with ground truth (GT) 3D object shapes and positions, and to demonstrate the accuracy and robustness of our method. All the experiments are conducted using an Intel(R) Core(TM) i7-9750H CPU@2.6GHZ, 16G memory, and Nvidia GTX 1080 Ti.

${ }^{1}$ https://www.opengl.org/ 
We define the following criteria for evaluation:

(1) $I o U_{2 D}$ : The intersection ratio of the GT to the $2 \mathrm{D}$ BBox of the projected quadric.

$$
I o U_{2 D}=\frac{B_{g t} \cap B_{\text {pred }}}{B_{g t} \cup B_{\text {pred }}}
$$

(2) $e_{\text {trans }}$ : The error of the GT quadric centroid translation to the predicted estimation, indicating the accuracy of the ellipsoid position.

$$
e_{\text {trans }}=\left\|t_{w q}^{g t}-t_{w q}^{\text {pred }}\right\|_{2}
$$

(3) $e_{\text {axe }}$ : The error of the GT ellipsoid axial length to the predicted estimation in the world coordinate, indicating the accuracy of the object shape.

$$
e_{\text {axe }}=\left\|a_{g t}-a_{\text {pred }}\right\|_{2}
$$

\section{A. Evaluation on Simulation Environment}

Simulation provides GT of object shapes and positions and it is easy to test the robustness of the compared methods with different types of disturbance. In the experiment, five cameras are evenly deployed within $18^{\circ}$ circular arcs to simulate the camera observation in the outdoor environment. An ellipsoid with varying shape and yaw rotation is deployed, the GT 2D object BBox and the position are provided. The yaw rotation of the ellipsoid is randomly sampled in the range of $\pm 5^{\circ}$ to simulate the object with rotation. To avoid the influence of random errors on the experimental results, for each type of noise, 10 ellipsoids are generated with Gaussian noise from 10 seeds resulting in a total of 100 trials.

To test the effect of different types of noise on the quadric initialization method, the relative camera poses are obtained by introducing zero-mean Gaussian noise with standard deviations in the range $5 \% \sim 30 \%$ to simulate the trajectory error. In addition, a detection BBox is simulated by adding the zero-mean Gaussian noise of $1 \% \sim 6 \%$ to the GT.

We compare methods of quadric initialization including (a) Nicholson et al [6] denoted as Q-SLAM, (b) Rubino et al [1] denoted as Conic-method, (c) the proposed method of separating quadric central translation without rotation assumption denoted as Tri, and (d) the proposed initialization method with rotation assumption denoted as Tri+Yaw.

Quantitative evaluation results of initialization methods with different types of noise are visualized in Fig.4, the plots show the trend of different evaluation criteria with the increase in noise, the performance of all methods degrades when noise increases.

It is obvious that the Q-SLAM method is the most sensitive to noise. When either the translation noise reaches $15 \%$, the rotation noise reaches $20 \%$, or the detection BBox noise reaches $2 \%$, Q-SLAM fails to construct ellipsoids, while the Conic-method maintains relatively good results under the effect of translation and rotation noise. However, the $e_{\text {trans }}$ and $e_{a x e}$ of the Conic-method increase rapidly with the increase of BBox noise, and it also fails to initialize the ellipsoids when the BBox noise exceeds $4 \%$. It can be seen that the error of our method remains stable under the translation and rotation noise, with the maximum axial error of $\mathbf{0 . 4 5} \mathbf{~ m}$ and maximum translation error of $0.89 \mathrm{~m}$. Also, these metrics are affected by the BBox noise in a small range, with the maximum axial error and translation error of $\mathbf{1 . 0 2} \mathbf{~ m}$ and $2.10 \mathrm{~m}$. These results show that our proposed method significantly improves the robustness of initialization with the minimal growth trend of noise.

The visualization results of quadric initialization are shown in Fig.3, where the red and the green ellipsoids are GT and estimation, respectively. Our proposed method outperforms all the compared methods.

TABLE I

SUCCESS RATE COMPARISON IN KITTI RAW DATA DATASET.

\begin{tabular}{c|ccc}
\hline Sequence & Ours & Conic [1] & Q-SLAM [6] \\
\hline 09 & $\mathbf{0 . 6 9 1 2}$ & 0.4468 & 0.2706 \\
22 & $\mathbf{0 . 6 5 1 2}$ & 0.3333 & 0.2923 \\
23 & $\mathbf{0 . 6 2 3 0}$ & 0.3949 & 0.2829 \\
36 & $\mathbf{0 . 6 0 4 7}$ & 0.4096 & 0.3514 \\
59 & $\mathbf{0 . 5 6 2 5}$ & 0.3556 & 0.2558 \\
93 & $\mathbf{0 . 4 8 1 5}$ & 0.2826 & 0.3617 \\
\hline Average & $\mathbf{0 . 6 0 2 3}$ & 0.3705 & 0.3024 \\
\hline
\end{tabular}

TABLE II

$I_{2 D}$ COMPARISON IN KITTI RAW DATA DATASET.

\begin{tabular}{c|ccc}
\hline Sequence & Ours & Conic [1] & Q-SLAM [6] \\
\hline 09 & $\mathbf{0 . 7 3 3 5}$ & 0.7252 & 0.7031 \\
22 & 0.7629 & $\mathbf{0 . 7 7 9 1}$ & 0.7662 \\
23 & 0.7509 & $\mathbf{0 . 7 5 2 9}$ & 0.6959 \\
36 & $\mathbf{0 . 7 6 0 4}$ & 0.7558 & 0.7127 \\
59 & 0.6508 & $\mathbf{0 . 6 8 7 8}$ & 0.6500 \\
93 & 0.7232 & 0.6751 & $\mathbf{0 . 7 4 3 3}$ \\
\hline Average & $\mathbf{0 . 7 3 0 3}$ & 0.7293 & 0.7119 \\
\hline
\end{tabular}

\section{B. Evaluation on KITTI Raw Data Dataset}

To evaluate the performance of the proposed method in outdoor environments, we select the KITTI Raw Data dataset [18] in particular the sequences $-09,-22,-23,-36,-59$, and -93 , which were recorded in urban and residential areas with vehicles. The dataset provides GT for vehicles, including 3DoF object size and 6-DoF object pose. With the extrinsic parameters of sensors, we can transform the object pose to camera coordinates.

Table I shows the success rate of initialization and ellipsoid reconstruction by different methods using different sequences. Tables II, III and IV show the experimental results of successfully constructed ellipsoids under different evaluation criteria.

From Table I, we can see that our method constructs ellipsoids for $\mathbf{6 0 . 2 \%}$ of the vehicles and reaches an increase of $\mathbf{6 2 . 6 \%}$ (from $37.0 \%$ to $60.23 \%$ ) and $\mathbf{9 9 . 2 \%}$ (from $30.24 \%$ to $60.23 \%$ ) in success rate compared with the Conic-method and the Q-SLAM, respectively, thus confirming the effectiveness of our initialization method. For the $I_{0} U_{2 D}$ metric, larger values indicate better reconstruction results. As can be seen from Table II, our method outperforms the other existing methods with respect to $I o U_{2 D}$ in sequence -09 and -36, with the overall best average of $\mathbf{7 3 . 0 3 \%}$. The compared methods give better results for individual sequences because they discard some detection results that fail to be initialized. For $e_{\text {trans }}$ and $e_{a x e}$, smaller values indicate better reconstruction 
TABLE III

TRANSLATION ERROR (M) COMPARISON IN KITTI RAW DATA DATASET.

\begin{tabular}{c|ccc}
\hline Sequence & Ours & Conic [1] & Q-SLAM [6] \\
\hline 09 & $\mathbf{2 . 5 4 5 6}$ & 2.7819 & 3.6110 \\
22 & 2.1769 & $\mathbf{1 . 9 5 5 2}$ & 1.9651 \\
23 & $\mathbf{2 . 3 3 4 1}$ & 5.6605 & 8.7088 \\
36 & $\mathbf{1 . 8 5 9 4}$ & 2.7175 & 6.9814 \\
59 & $\mathbf{1 . 3 2 7 6}$ & 1.6883 & 1.3874 \\
93 & $\mathbf{2 . 5 2 2 6}$ & 5.4130 & 4.0654 \\
\hline Average & $\mathbf{2 . 1 2 7 7}$ & 3.3694 & 4.4532 \\
\hline
\end{tabular}

TABLE IV

AXIAL LENGTH ERROR (M) COMPARISON IN KITTI RAW DATA DATASET.

\begin{tabular}{c|ccc}
\hline Sequence & Ours & Conic [1] & Q-SLAM [6] \\
\hline 09 & $\mathbf{0 . 6 2 7 1}$ & 1.2618 & 1.1400 \\
22 & $\mathbf{0 . 5 5 6 5}$ & 0.7233 & 0.8356 \\
23 & $\mathbf{0 . 5 4 9 4}$ & 1.7886 & 0.6837 \\
36 & $\mathbf{0 . 7 1 2 1}$ & 1.2797 & 0.8799 \\
59 & $\mathbf{0 . 6 7 0 6}$ & 1.8467 & 1.2908 \\
93 & $\mathbf{0 . 7 3 5 7}$ & 1.3156 & 0.8574 \\
\hline Average & $\mathbf{0 . 6 4 1 9}$ & 1.3693 & 0.9479 \\
\hline
\end{tabular}

results. As can be seen from Table III and Table IV, our method outperforms the compared methods in all cases except for sequence-22, with the average ellipsoid central translation error of $\mathbf{2 . 1 2 7} \mathrm{m}$, nearly $\mathbf{5 2 . 2 \%}$ reduction in error. In addition, our average axial length error is $0.642 \mathbf{~ m}$, a $\mathbf{5 0 . 8 \%}$ reduction in error, compared with $1.369 \mathrm{~m}$ and 0.947 $\mathrm{m}$ for the other techniques. These experimental results show the robustness and accuracy of the proposed method for quadric reconstruction in outdoor scenes.

We show the reconstructed quadrics in Fig.1. The yellow quadrics illustrate the accurate orientation and shape of the estimated ellipsoids when projected onto the image frame. The magenta lines show the center of the ellipsoids in previous frames projected onto the current image frame, demonstrating the accuracy of data association with the ODA algorithm.

\section{Effect of quadrics on localization}

The quantitative results of localization on the KITTI Raw Data dataset can be found in Table V. The RMSE of the absolute translation error (ATE) is adopted as evaluation metrics [22]. These results show a great improvement of the proposed system in the performance of localization compared with QSLAM in all compared cases. Meanwhile, compared with the optimization without quadrics, the joint BA optimization improves the localization accuracy by the average of $\mathbf{8 \%}$ on sequence-09, -27, and -36 with $1.09 \mathrm{~m}, \mathbf{1 . 0 8} \mathbf{~ m}$ and $\mathbf{4 . 2 5}$ $\mathbf{m}$, respectively, while a slight decrease in performance on sequence-22 and -23 with $\mathbf{1 . 7 6} \mathbf{~ m}$ and $\mathbf{0 . 6 1} \mathbf{~ m}$, respectively. The performance loss of Q-SLAM is caused by the joint optimization with the false associated and error quadric landmarks construction, and the detected object occlusions and moving objects in the scenarios (e.g., sequence-22 and -23) are composed of a lot of moving cars, which create false BBoxes and negatively impact the estimated trajectory of the proposed system in outdoor environments.

The results show the localization accuracy of the proposed system, and the joint optimization with accurate objects
TABLE V

RMS ATE (M) ON THE KITTI RAW DATA DATASETS.

\begin{tabular}{c|ccccc}
\hline Sequence & -09 & -22 & -23 & -27 & -36 \\
\hline BA with quadrics & $\mathbf{1 . 0 9}$ & 1.82 & 0.64 & $\mathbf{1 . 0 8}$ & $\mathbf{4 . 2 5}$ \\
BA w/o quadrics & 1.11 & $\mathbf{1 . 7 6}$ & $\mathbf{0 . 6 1}$ & 1.18 & 4.57 \\
Q-SLAM & 5.73 & 4.32 & 2.10 & 7.91 & 14.74 \\
\hline
\end{tabular}
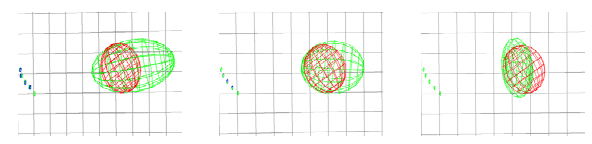

Conic-method
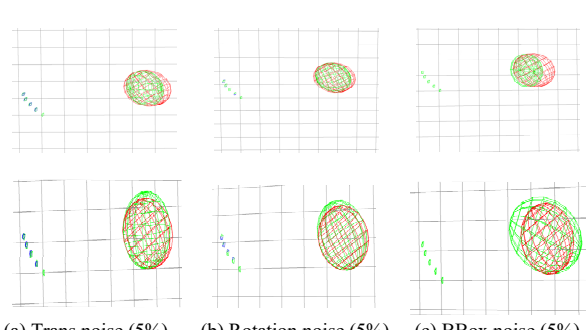

(a) Trans noise $(5 \%)$

(c) BBox noise $(5 \%)$

Fig. 3. The visualization results of initialization performance of methods to different types of noise. Our method outperform the compared techniques.

estimation can improve localization accuracy to some extent. In future work, the accuracy and robustness of dynamic objects association and the reduction of their impact will be explored.

\section{Real-time performance evaluation}

Table VI summarizes the run time for each image frame processed by the main modules presenting the mean value and its corresponding range, showing that our proposed system is able to run in real-time with $10 \mathbf{~ H Z}$. The detection thread takes an average of $\mathbf{5 6 . 3 4} \mathbf{~ m s}$ to process the frame's segmentation. Time for tracking thread takes approximately $100 \mathrm{~ms}$ with the efficient ODA and SQP algorithms only taking an average of $\mathbf{1 . 5 1} \mathbf{~ m s}$ and $\mathbf{0 . 1 7} \mathbf{~ m s}$, respectively, while the ORB extraction process consumes most of the time, nonetheless this still ensures real-time pose estimation performance of $10 \mathrm{HZ}$. For the local mapping thread, the local BA with quadrics takes an average of $\mathbf{2 1 1 . 1 4} \pm \mathbf{9 2 . 5 6}$ $\mathbf{m s}$, and the total computing time of the thread is $\mathbf{3 1 8} \pm \mathbf{1 0 3 . 1 6}$ ms depending on the number of associated quadrics to be optimized. As both the operations are processed in parallel in separate threads as Fig.2 shows, they do not interfere with the real-time performance of the rest of the system.

\section{CONCLUSION}

In this work, a novel pipeline of real-time object SLAM with 3D quadric landmarks is presented. A quadric initialization method based on the SQP algorithm is proposed to improve the robustness and accuracy of ellipsoid reconstruction. The data association is solved by the ODA algorithm which ensures highly accurate object pose estimation and ellipsoid landmark representation. Extensive experiments are conducted to show that the proposed system is accurate and robust to observation noise in object SLAM and significantly outperforms other methods in an outdoor environment. 

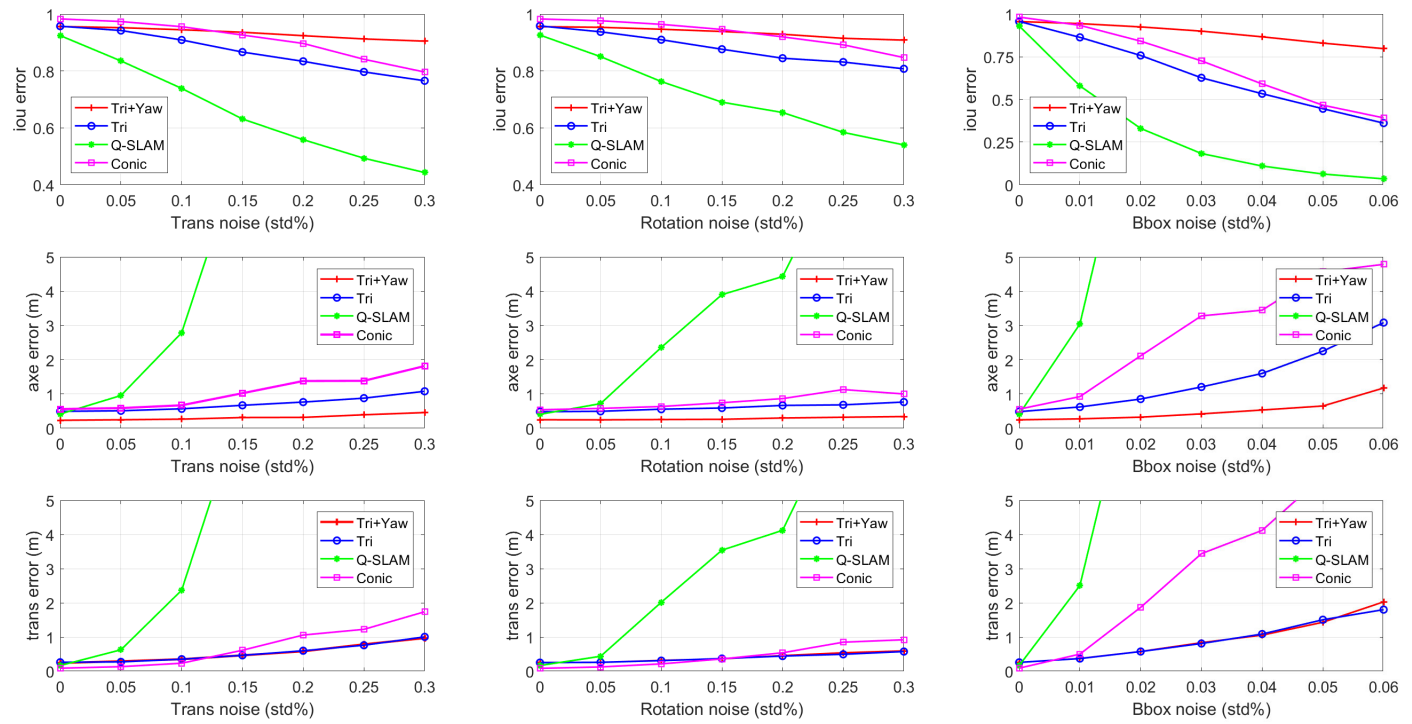

Fig. 4. The quadric initialization performance of compared methods to different types of observation noise on the simulation.

TABLE VI

RUNNING TIME (MS) OF THE MAIN MODULES OF THE PROPOSED SYSTEM ON THE KITTI RAW DATA DATASET.

\begin{tabular}{|c|c|c|c|c|c|}
\hline \multirow{4}{*}{ Settings } & Sequence & -09 & -22 & -23 & -36 \\
\hline & Resolution & $1242 \times 375$ & $1242 \times 375$ & $1242 \times 375$ & $1242 \times 375$ \\
\hline & Camera FPS & $10 \mathrm{~Hz}$ & $10 \mathrm{~Hz}$ & $10 \mathrm{~Hz}$ & $10 \mathrm{~Hz}$ \\
\hline & ORB Features & 2000 & 2000 & 2000 & 2000 \\
\hline Detection & Segmentation & $57.61 \pm 6.75$ & $53.51 \pm 5.69$ & $59.86 \pm 5.56$ & $54.36 \pm 6.38$ \\
\hline \multirow{4}{*}{ Tracking } & ORB Extraction & $59.67 \pm 7.93$ & $59.15 \pm 8.37$ & $60.99 \pm 8.84$ & $56.94 \pm 8.25$ \\
\hline & ODA & $2.04 \pm 3.34$ & $0.94 \pm 0.77$ & $2.12 \pm 0.78$ & $0.93 \pm 1.01$ \\
\hline & SQP & $0.17 \pm 0.21$ & $0.24 \pm 0.15$ & $0.14 \pm 0.12$ & $0.11 \pm 0.31$ \\
\hline & Total time & $90.14 \pm 8.47$ & $86.61 \pm 9.31$ & $93.3 \pm 10.74$ & $85.88 \pm 9.12$ \\
\hline \multirow{2}{*}{ Local Mapping } & Local BA & $220.06 \pm 119.05$ & $211.92 \pm 93.86$ & $204.23 \pm 68.10$ & $208.33 \pm 89.22$ \\
\hline & Total time & $327.29 \pm 126.71$ & $314.45 \pm 114.46$ & $321.18 \pm 79.24$ & $309.98 \pm 92.21$ \\
\hline
\end{tabular}

In further work, we will explore finding the semantic relationships between object ellipsoids, and using the semantic information of the object map to perform re-localization.

\section{REFERENCES}

[1] C. Rubino, M. Crocco, and A. Del Bue, "3d object localisation from multi-view image detections," IEEE transactions on pattern analysis and machine intelligence, vol. 40, no. 6, pp. 1281-1294, 2017.

[2] K. Ok, K. Liu, K. Frey, J. P. How, and N. Roy, "Robust object-based slam for high-speed autonomous navigation," in 2019 International Conference on Robotics and Automation (ICRA). IEEE, 2019, pp. 669-675.

[3] S. Yang and S. Scherer, "Monocular object and plane slam in structured environments," 2018.

[4] — - "Cubeslam: Monocular 3-d object slam," IEEE Transactions on Robotics, vol. 35, no. 4, pp. 925-938, 2019.

[5] Y. Wu, Y. Zhang, D. Zhu, Y. Feng, S. Coleman, and D. Kerr, "Eaoslam: Monocular semi-dense object slam based on ensemble data association," in 2020 IEEE/RSJ International Conference on Intelligent Robots and Systems (IROS). IEEE, 2020, pp. 4966-4973.

[6] L. Nicholson, M. Milford, and N. Sünderhauf, "Quadricslam: Dual quadrics from object detections as landmarks in object-oriented slam," IEEE Robotics and Automation Letters, vol. 4, no. 1, pp. 1-8, 2018.

[7] Z. Liao, W. Wang, X. Qi, X. Zhang, and R. Wei, "Object-oriented slam using quadrics and symmetry properties for indoor environments," 2020.

[8] V. Gaudillière, G. Simon, and M. O. Berger, "Perspective-2-ellipsoid: Bridging the gap between object detections and 6-dof camera pose," IEEE Robotics and Automation Letters, vol. 5, no. 4, pp. 5189-5196, 2020.

[9] M. Hosseinzadeh, K. Li, Y. Latif, and I. Reid, "Real-time monocular object-model aware sparse slam," in 2019 International Conference on Robotics and Automation (ICRA). IEEE, 2019, pp. 7123-7129.
[10] L. Xia, J. Cui, R. Shen, X. Xu, and X. Li, "A survey of image semantics-based visual simultaneous localization and mapping: Application-oriented solutions to autonomous navigation of mobile robots," International Journal of Advanced Robotic Systems, vol. 17, no. 3, p. 172988142091918, 2020.

[11] J. Redmon and A. Farhadi, "Yolov3: An incremental improvement," arXiv preprint arXiv:1804.02767, 2018.

[12] D. Bolya, C. Zhou, F. Xiao, and Y. J. Lee, "Yolact: Real-time instance segmentation," in Proceedings of the IEEE/CVF International Conference on Computer Vision, 2019, pp. 9157-9166.

[13] K. He, G. Gkioxari, P. Dollár, and R. Girshick, "Mask r-cnn," in Proceedings of the IEEE international conference on computer vision, 2017, pp. 2961-2969.

[14] R. F. Salas-Moreno, R. A. Newcombe, H. Strasdat, P. H. Kelly, and A. J. Davison, "Slam++: Simultaneous localisation and mapping at the level of objects," in Proceedings of the IEEE conference on computer vision and pattern recognition, 2013, pp. 1352-1359.

[15] G. Cross and A. Zisserman, "Quadric reconstruction from dual-space geometry," in Sixth International Conference on Computer Vision (IEEE Cat. No. 98CH36271). IEEE, 1998, pp. 25-31.

[16] Z. Qian, K. Patath, J. Fu, and J. Xiao, "Semantic slam with autonomous object-level data association," in 2021 IEEE international conference on robotics and automation (ICRA). IEEE, 2021.

[17] H. W. Kuhn, "The hungarian method for the assignment problem," Naval research logistics quarterly, vol. 2, no. 1-2, pp. 83-97, 1955.

[18] A. Geiger, P. Lenz, C. Stiller, and R. Urtasun, "Vision meets robotics: The kitti dataset," International Journal of Robotics Research (IJRR), 2013.

[19] C. Campos, R. Elvira, J. Rodríguez, J. Montiel, and J. Tardós, "Orbslam3: An accurate open-source library for visual, visual-inertial and multi-map slam," 2020

[20] R. Tian, Y. Zhang, D. Zhu, S. Liang, S. Colman, and D. Kerr, "Accurate and robust scale recovery for monocular visual odometry based on plane geometry," in 2021 IEEE international conference on robotics and automation (ICRA). IEEE, 2021. 
[21] A. Bewley, Z. Ge, L. Ott, F. Ramos, and B. Upcroft, "Simple online and realtime tracking," in 2016 IEEE International Conference on Image Processing (ICIP), 2016.

[22] M. Grupp, "evo: Python package for the evaluation of odometry and slam." https://github.com/MichaelGrupp/evo, 2017. 